for $A P O E \varepsilon 2$, which represents only $0.2 \%$ of the autopsy-confirmed sample. Furthermore, pathology-confirmed AD represents $29 \%$ of the $A P O E \& 2 / \varepsilon 2$ patients diagnosed with dementia. Conclusions: Although rare, autopsy-confirmed $\mathrm{AD}$ can be present in $A P O E$ $\varepsilon 2 / \varepsilon 2$ carriers and, most importantly, was seen more frequently than previously believed.

\section{P3-457 COMBINED NEUROPATHOLOGICAL PATHWAYS ACCOUNT FOR AGE-RELATED INCREASES IN RISK OF DEMENTIA}

Melinda C. Power ${ }^{1}$, Beth C. Mormino ${ }^{2}$, Anja Soldan ${ }^{3}$, Bryan D. James ${ }^{4,5}$, Lei Yu ${ }^{6}$, Nicole Armstrong ${ }^{7}$, Katherine J. Bangen ${ }^{8}$, Lisa Delano-Wood ${ }^{8}$, Melissa Lamar ${ }^{9}$, Yen Lim ${ }^{10}$, Kelly N. H. Nudelman ${ }^{11}$, Laura B. Zahodne ${ }^{12}$, Alden L. Gross ${ }^{7}$, Dan Mungas ${ }^{13}$, Keith F. Widaman ${ }^{14}$, Julie A. Schneider ${ }^{6},{ }^{1}$ George Washington University, Washington, D.C., USA ${ }^{2}$ Massachusetts General Hospital, Boston, MA, USA; ${ }^{3}$ Johns Hopkins University School of Medicine, Baltimore, MD, USA; ${ }^{4}$ Rush University, Chicago, IL, USA; ${ }^{5}$ Rush Alzheimer's Disease Center, Chicago, IL, USA; ${ }^{6}$ Rush University Medical Center, Chicago, IL, USA; ${ }^{7}$ Johns Hopkins Bloomberg School of Public Health, Baltimore, MD, USA; ${ }^{8}$ University of California San Diego, La Jolla, CA, USA; ${ }^{9}$ Rush Alzheimer's Disease Center, Rush University Medical Center, Chicago, IL, USA; ${ }^{10}$ The Florey Institute of Neuroscience and Mental Health, Melbourne, Australia; ${ }^{11}$ Indiana University-Purdue University, Indianapolis, IN, USA; ${ }^{12}$ University of Michigan, Ann Arbor, MI, USA;

${ }^{13}$ University of California, Davis, Sacramento, CA, USA;

${ }^{14}$ University of California, Riverside, Riverside, CA, USA. Contact e-mail:power@gwu.edu

Background:Our objectives were to characterize the interrelationship of known dementia-related neuropathologies in one comprehensive model and to quantify the extent to which the accumulation of neu-

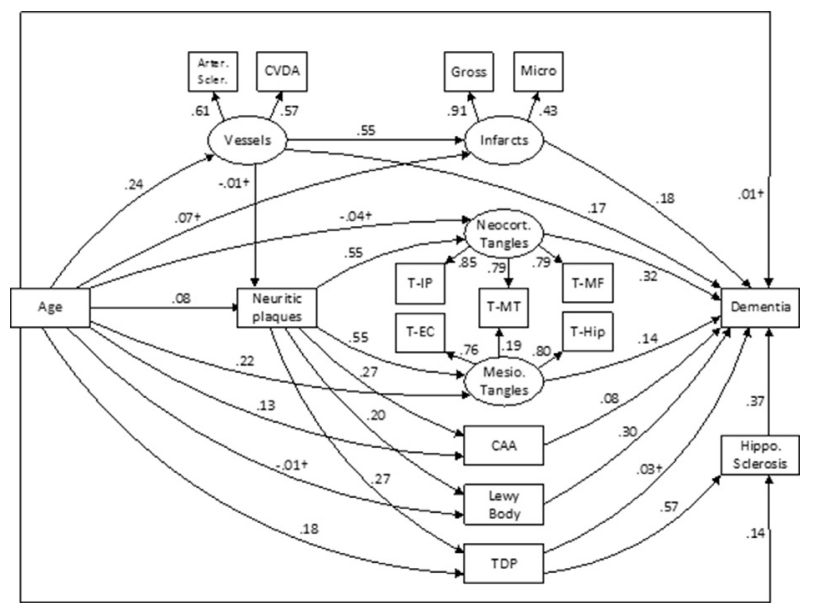

Figure 3. Results of path analysis of combined pathologic pathways mediating the effect of age on dementia risk. Numbers are standardized coefficients or factor loadings. 'denotes non-significant paths. Abbreviations: Arter. Scler: arteriolosclerosis, CAA: cerebral amyloid angiopathy; CVDA: atherosclerosis, Gross: gross infarcts, Hippo. Sclerosis: hippocampal sclerosis; Micro: microinfarcts; Mesio. Tangles: mesiotemporal neurofibrilary tangles; Neocort Tangles: neocortical neurofibrilary tangles, TDP: TAR DNA- binding protein 43, T-EC: neurofibrillary tangles in the entorhinal cortex, T-Hip: neurofibrillary tangles in the hippocampus, T-IP: neurofibrillary tangles in the inferior parietal cortex, T-MF: neurofibrillary tangles in the midfrontal cortex, T-MT: neurofibrillary tangles in the midtemporal cortex. ropathologies accounts for the association between age and dementia. Methods: We used data from 1,362 autopsied participants of three community-based clinico-pathologic cohorts: the Religious Orders Study, the Rush Memory and Aging Project, and the Minority Aging Research Study. We estimated a series of structural equation models summarizing a priori hypothesized neuropathological pathways between age and dementia risk individually and collectively. Results: At the time of death (mean age: 89 years), $44 \%$ of our sample had a clinical dementia diagnosis. Of those with a clinical dementia diagnosis, $85 \%$ were diagnosed with Alzheimer's disease dementia without a second cause of cognitive impairment. When considered individually, our vascular, amyloid/tau, neocortical Lewy body, and TAR DNA-binding protein 43 (TDP-43)/hippocampal sclerosis pathways each accounted for a substantial proportion of the association between age and dementia. When considered collectively, the four pathways fully accounted for all variance in dementia risk previously attributable to age. Pathways involving amyloid/tau, neocortical Lewy bodies, and TDP-43/hippocampal sclerosis were interdependent, as amyloid beta plaques were important in all three. The importance of the pathways varied, with the vascular pathway accounting for $32 \%$ of the association between age and dementia, while the remaining three interrelated pathways together accounted for $68 \%$ (amyloid/tau: 24\%, Lewy body: 1\%, and TDP-43/hippocampal sclerosis: $43 \%$ ). Conclusions: Age-related increases in dementia risk can be attributed to accumulation of multiple pathologies, each of which independently contributes to dementia risk. Multipronged approaches may be necessary if we are to develop effective therapies. This finding may also have implications for the traditional notions of "normal" cognitive aging.

\section{P3-458 CLINICOPATHOLOGIC DIFFERENCES WITHIN ALZHEIMER'S DISEASE CASES FROM THE FLORIDA AUTOPSIED MULTI-ETHNIC (FLAME) STUDY}

Octavio A. Santos ${ }^{1}$, Ranjan Duara ${ }^{2}$, John A. Lucas ${ }^{3}$, Maria T. GreigCusto $^{4}$, Amanda M. Liesinger ${ }^{5}$, Fadi S. Hanna Al-Shaikh ${ }^{5}$, Owen A. Ross ${ }^{5}$, Nilufer Ertekin-Taner ${ }^{5}$, Otto Pedraza ${ }^{5}$, Neill R. Graff-Radford ${ }^{5}$, Dennis W. Dickson ${ }^{5}$, Melissa E. Murray ${ }^{6},{ }^{1}$ Mayo Clinic, Jacksonville, $F L$, USA; ${ }^{2}$ Mount Sinai Medical Center, Miami, FL, USA; ${ }^{3}$ Mayo Clinic, Jacksonville, FL, USA; ${ }^{4}$ Mount Sinai Medical Center, Miami Beach, FL, USA; ${ }^{5}$ Mayo Clinic, Jacksonville, FL, USA; ${ }^{6}$ Mayo Clinic Florida, Jacksonville, FL, USA. Contact e-mail: Santos.Octavio@mayo.edu

Background: Despite the projected increase in dementia prevalence and growing proportion of ethnic minorities in the U.S., little is known about clinicopathologic differences in ethnoracially diverse individuals with autopsy-confirmed Alzheimer's disease (AD). Of particular interest is the applicability of the "Hispanic mortality paradox" in the context of AD. This epidemiological phenomenon has previously suggested comparable or more advantaged longevity despite socioeconomic disadvantage relative to Caucasians/nonHispanic Whites. Our study sought to examine demographic risk variables for $\mathrm{AD}$ dementia, clinical progression, and neuropathologic outcomes within the Florida Autopsied Multi-Ethnic (FLAME) cohort. Methods: Individuals from the FLAME study were grouped according to their self-reported ethnoracial status as Hispanic $(n=67)$, African-American/Black $(n=19)$, and Caucasian/White non-Hispanic $(n=1539)$. Clinical history was reviewed to abstract antemortem information (see Table), including occupation that was converted to a 6-point scale ranging from 0 (no job) to 6 (highly skilled). The proportion of individuals surviving from age 